Published in final edited form as:

Nature. 2017 June 21; 546(7659): E4-E5. doi:10.1038/nature22085.

\title{
Zanos et al. reply
}

Panos Zanos ${ }^{1}$, Ruin Moaddel ${ }^{2}$, Patrick J. Morris ${ }^{3}$, Polymnia Georgiou ${ }^{1}$, Jonathan Fischell ${ }^{4}$, Greg I. Elmer ${ }^{1,5,6}$, Manickavasagom Alkondon ${ }^{7}$, Peixiong Yuan ${ }^{8}$, Heather J. Pribut ${ }^{1}$, Nagendra S. Singh ${ }^{2}$, Katina S. S. Dossou ${ }^{2}$, Yuhong Fang ${ }^{3}$, Xi-Ping Huang $^{9}$, Cheryl L. Mayo $^{6}$, Edson X. Albuquerque ${ }^{5,7,10}$, Scott M. Thompson ${ }^{1,4}$, Craig J. Thomas ${ }^{3}$, Carlos A. Zarate $\mathrm{Jr}^{8}$, and Todd D. Gould ${ }^{1,5,11}$

${ }^{1}$ Department of Psychiatry, University of Maryland School of Medicine, Baltimore, Maryland 21201, USA

2Biomedical Research Center, National Institute on Aging, National Institutes of Health, Baltimore, Maryland 21224, USA

${ }^{3}$ Division of Preclinical Innovation, National Center for Advancing Translational Sciences, National Institutes of Health, Rockville, Maryland 20892, USA

${ }^{4}$ Department of Physiology, University of Maryland School of Medicine, Baltimore, Maryland 21201, USA

${ }^{5}$ Department of Pharmacology, University of Maryland School of Medicine, Baltimore, Maryland 21201, USA

${ }^{6}$ Maryland Psychiatric Research Center, University of Maryland School of Medicine, Baltimore, Maryland 21228, USA

${ }^{7}$ Department of Epidemiology and Public Health, Division of Translational Toxicology, University of Maryland School of Medicine, Baltimore, Maryland 21201, USA

${ }^{8}$ Experimental Therapeutics and Pathophysiology Branch, Intramural Research Program, National Institute of Mental Health, National Institutes of Health, Bethesda, Maryland 20814, USA

${ }^{9}$ NIMH Psychoactive Drug Screening Program, Department of Pharmacology and Division of Chemical Biology and Medicinal Chemistry, University of North Carolina Chapel Hill Medical School, Chapel Hill, North Carolina 27516, USA

${ }^{10}$ Department of Medicine, University of Maryland School of Medicine, Baltimore, Maryland 21201, USA

${ }^{11}$ Department of Anatomy and Neurobiology, University of Maryland School of Medicine, Baltimore, Maryland 21201, USA

In the accompanying Comment ${ }^{1}$, Suzuki et al. confirmed our previous findings ${ }^{2}$ that the ketamine metabolite $(2 R, 6 R)$-hydroxynorketamine (HNK) does not functionally inhibit the NMDAR at concentrations relevant to its antidepressant actions recently reported in mice (that is, approximately $10 \mu \mathrm{M})$. We reported that $10 \mu \mathrm{M}(2 R, 6 R)-\mathrm{HNK}$, which equates to the maximum concentration of brain exposure $(10.69 \mu \mathrm{M}$ (ref. 2)) after intraperitoneal administration of a $10 \mathrm{mg} \mathrm{kg}^{-1}$ antidepressant dose of $(2 R, 6 R)$-HNK in mice, robustly potentiated excitatory postsynaptic potentials in hippocampal slices without functionally 
inhibiting the $\mathrm{NMDAR}^{2}$, as Suzuki et al. now confirm. Unless NMDAR inhibition is observed at $10 \mu \mathrm{M}(2 R, 6 R)-\mathrm{HNK}$, it is probably not of major relevance to the antidepressant actions of $(2 R, 6 R)$-HNK. Notably, $(2 \mathrm{R}, 6 \mathrm{R})$-HNK administration also resulted in significant antidepressant actions at the dose of $5 \mathrm{mg} \mathrm{kg}-^{1}$ in mice, which would produce even lower concentrations of the metabolite in the brain than $10 \mu \mathrm{M}$ (ref. 2). Furthermore, our fieldpotential electrophysiology experiments were conducted in the presence of the NMDAR antagonist AP5 $(80 \mu \mathrm{M})$, demonstrating that the AMPAR-mediated synaptic potentiation we observed is independent of any capacity of $(2 R, 6 R)$-HNK to inhibit the NMDAR.

We agree with Suzuki et al. that assessing higher concentrations of $(2 R, 6 R)$-HNK on NMDAR-mediated miniature excitatory postsynaptic currents (NMDAR-mEPSCs) responses might be important in providing information for off-target effects of this metabolite, and their study indeed provides evidence for a modest inhibition $(\sim 37 \%)$ at the concentration of $50 \mu \mathrm{M}$. The absence of $\mathrm{Mg}^{2+}$ in the testing conditions may influence the apparent discrepancy between $37 \%$ inhibition of NMDAR-mEPSCs at the $50 \mu \mathrm{M}$ concentration (for example, as is the case with memantine, which only inhibits NMDARmEPSCs in the absence of $\mathrm{Mg}^{2+}$, and does not have antidepressant properties in humans ${ }^{3}$ ), and the previously reported lack of displacement of $\left[{ }^{3} \mathrm{H}\right] \mathrm{MK}-801$ binding at less than 100 $\mu \mathrm{M}$ (ref. 4). It will be important to follow up on this finding with a full concentration response curve in order to determine the half maximal inhibitory concentration $\left(\mathrm{IC}_{50}\right)$ of $(2 R, 6 R)$-HNK on NMDAR-mEPSC responses, and to compare to the $\mathrm{IC}_{50}$ of $(R, S)$ ketamine, under the same conditions using physiological levels of $\mathrm{Mg}^{2+}$. Notably, we did not observe NMDAR-inhibition-associated psychostimulant, self-administration, drug discrimination and pre-pulse inhibition side effects of $(2 R, 6 R)$-HNK at any dose, including up to $375 \mathrm{mg} \mathrm{kg}^{-1}$ (ref. 2).

The authors also observed decreased phosphorylation of the eukaryotic elongation factor 2 (eEF2) only at the concentration of $50 \mu \mathrm{M}$. Although this is the same high concentration at which $(2 R, 6 R)$-HNK induced an inhibition of the NMDAR responses, these data do not provide direct evidence of a functional link between the moderate inhibition of NMDARs and the decrease in eEF2 phosphorylation. Indeed there are multiple mechanisms other than NMDAR inhibition that could be responsible for the observed eEF2 dephosphorylation ${ }^{5-8}$. It is also important to note that although direct application of ketamine ${ }^{9}$ or the alternative NMDAR blocker MK-801 (ref. 10) to cultured neurons or hippocampal slices was previously reported to induce NMDAR-inhibition-dependent synaptic potentiation, MK-801 has repeatedly failed to induce ketamine-like sustained antidepressant effects in several animal tests ${ }^{2,9,11,12}$. It is therefore probable that synaptic strengthening as a consequence of NMDAR inhibition (proposed by Suzuki et al.) is not solely responsible for the antidepressant actions of ketamine. Indeed, the results of clinical trials indicate that several other NMDAR antagonists lack the full antidepressant actions of ketamine in humans ${ }^{13}$.

Suzuki et al. also propose that NMDAR inhibition by $(R, S)$-ketamine is responsible for the initiation of antidepressant responses (that is, the acute actions), and HNK metabolites mediate the long-term antidepressant effects of ketamine through continued NMDAR inhibition. This hypothesis is not supported by our pharmacokinetic measurements of ketamine and its metabolites in the brain of mice following intraperitoneal administration of 
$(R, S)$-ketamine, which show that both ketamine and its metabolites peak at similar early time points, have a similar short half-life, and are below detectable levels by four hours after treatment ${ }^{2}$.

In conclusion, the data presented by Suzuki et al. further support an NMDAR-inhibitionindependent mechanism of antidepressant action of the ketamine metabolite $(2 R, 6 R)$-HNK at physiologically relevant concentrations. Although development of $(2 R, 6 R)-\mathrm{HNK}$ as an anti depressant drug, which does not induce NMDAR-mediated side effects, can proceed independently of target identification, it remains important to identify its primary pharmacological target at anti depressant-relevant concentrations $(\sim 10 \mu \mathrm{M})$.

Author Irving W. Wainer has chosen not to be listed on this Reply.

\section{References}

1. Suzuki, K., Nosyreva, E., Hunt, KW., Kavalali, ET., Monteggia, LM. Effects of a ketamine metabolite on synaptic NMDAR function; Nature. 2017. p. 546http://dx.doi.org/10.1038/ nature 22084

2. Zanos P, et al. NMDAR inhibition-independent antidepressant actions of ketamine metabolites. Nature. 2016; 533:481-486. [PubMed: 27144355]

3. Gideons ES, Kavalali ET, Monteggia LM. Mechanisms underlying differential effectiveness of memantine and ketamine in rapid antidepressant responses. Proc Natl Acad Sci USA. 2014; 111:8649-8654. [PubMed: 24912158]

4. Moaddel $\mathrm{R}$, et al. Sub-anesthetic concentrations of $(R, S)$-ketamine metabolites inhibit acetylcholineevoked currents in a 7 nicotinic acetylcholine receptors. Eur J Pharmacol. 2013; 698:228-234. [PubMed: 23183107]

5. Hizli AA, et al. Phosphorylation of eukaryotic elongation factor 2 (eEF2) by cyclin A-cyclindependent kinase 2 regulates its inhibition by eEF2 kinase. Mol Cell Biol. 2013; 33:596-604. [PubMed: 23184662]

6. Knebel A, Morrice N, Cohen P. A novel method to identify protein kinase substrates: eEF2 kinase is phosphorylated and inhibited by SAPK4/p386. EMBO J. 2001; 20:4360-4369. [PubMed: 11500363]

7. Redpath NT, Foulstone EJ, Proud CG. Regulation of translation elongation factor-2 by insulin via a rapamycin-sensitive signalling pathway. EMBO J. 1996; 15:2291-2297. [PubMed: 8641294]

8. Wang X, et al. Regulation of elongation factor 2 kinase by p90(RSK1) and p70 S6 kinase. EMBO J. 2001; 20:4370-4379. [PubMed: 11500364]

9. Autry AE, et al. NMDA receptor blockade at rest triggers rapid behavioural antidepressant responses. Nature. 2011; 475:91-95. [PubMed: 21677641]

10. Nosyreva E, et al. Acute suppression of spontaneous neurotransmission drives synaptic potentiation. J Neurosci. 2013; 33:6990-7002. [PubMed: 23595756]

11. Maeng S, et al. Cellular mechanisms underlying the antidepressant effects of ketamine: role of alpha-amino-3-hydroxy-5-methylisoxazole-4-propionic acid receptors. Biol Psychiatry. 2008; 63:349-352. [PubMed: 17643398]

12. Yang B, Ren Q, Ma M, Chen QX, Hashimoto K. Antidepressant effects of (+)-MK-801 and (-)MK-801 in the social defeat stress model. Int J Neuropsychopharmacol. 2016; 19:pyw080. [PubMed: 27608811]

13. Newport DJ, et al. Ketamine and other NMDA antagonists: early clinical trials and possible mechanisms in depression. Am J Psychiatry. 2015; 172:950-966. DOI: 10.1038/nature22085 [PubMed: 26423481] 